\title{
Cover times of random searches
}

\author{
Marie Chupeau', Olivier Bénichou ${ }^{1 \star}$ and Raphaël Voituriez ${ }^{1,2 \star}$
}

\begin{abstract}
How long must one undertake a random search to visit all sites of a given domain? This time, known as the cover time', is a key observable to quantify the efficiency of exhaustive searches, which require a complete exploration of an area and not only the discovery of a single target. Examples range from immune-system cells chasing pathogens ${ }^{2}$ to animals harvesting resources ${ }^{3,4}$, from robotic exploration for cleaning or demining to the task of improving search algorithms ${ }^{5}$. Despite its broad relevance, the cover time has remained elusive and so far explicit results have been scarce and mostly limited to regular random walks ${ }^{6-9}$. Here we determine the full distribution of the cover time for a broad range of random search processes, including Lévy strategies ${ }^{10-14}$, intermittent strategies ${ }^{4,15,16}$, persistent random walks ${ }^{17}$ and random walks on complex networks ${ }^{18}$, and reveal its universal features. We show that for all these examples the mean cover time can be minimized, and that the corresponding optimal strategies also minimize the mean search time for a single target, unambiguously pointing towards their robustness.
\end{abstract}

Random search processes have been proved over recent years to be involved in a broad range of contexts at various scales, from the search for specific sequences on DNA by proteins to animal foraging ${ }^{3,4}$. So far, the main tools to quantify the efficiency of such search processes could be expressed in terms of the time needed for the searcher to reach a single target, the so-called first-passage time $\mathrm{e}^{18-22}$. However, as soon as several targets need to be found, which is a recurrent situation in chemistry, ecology, or robotics, the relevant observable is rather the time needed to reach a fraction of the domain sites (see Fig. 1). The extreme case of such exhaustive searches where all sites of a domain need to be visited defines the so-called cover time, which is of particular interest as it gives the time needed to find all targets of a domain with certainty; its determination is a long-standing problem of random walk theory $y^{1,23}$ (see refs 24,25 for related observables).

Nevertheless, analytical results on cover times are scarce. Important steps were achieved in ref. 26, where the mean cover time of an interval was analytically calculated for one-dimensional symmetric nearest-neighbour random walks, both for periodic and reflecting boundary conditions. In dimensions greater or equal to three, Aldous ${ }^{23}$ has determined the leading behaviour of the mean cover time in the limit of large domain size, which was reproduced by numerical simulations in ref. 27. In the physics literature, these results have been extended to the two-dimensional case in ref. 6 , which has since then been refined in the mathematics literature $e^{7-9}$. Notably, all these results were so far essentially limited to the case of regular random walks-that is, symmetric nearest-neighbour random walks in Euclidean geometries.

However, recently, several classes of more complex random search strategies, including Lévy strategies ${ }^{10-14}$, intermittent strategies $^{4,15,16}$ and persistent random walks ${ }^{17}$, have emerged and been shown theoretically to be efficient (see Fig. 1). In this context, existing theoretical studies have up to now focused mainly on the first-passage time to a single target, and the cover time of these random search processes, required to quantify the efficiency of exhaustive searches, has been left aside. The analytical determination of the entire distribution of cover time type observables for general classes of random walks, including the above, is at the core of this paper.

We consider a random walker moving on a network of $N$ sites. The random walk is assumed to be Markovian and noncompact (that is, transient: in infinite space, the probability for the walker to ever reach a given site is strictly smaller than one ${ }^{28}$ ). This covers a large class of processes relevant to search problems. We denote by $\tau(M, N)$ the partial cover time, defined as the time needed to visit any $M$ distinct sites of the network. The alternative problem of determining the time needed to visit $M$ given sites (chosen at random), called the random cover time ${ }^{27}$, will be addressed below. Note that taking $M=N$ (for both the partial and the random cover time) yields the full cover time, to which most of the literature has been devoted so far. In practice, we will focus on large values of $M, N$. The starting point is to introduce $\theta(N-k, N) \equiv \tau(k+1, N)-\tau(k, N)$, defined as the time needed for the number of distinct sites visited by the random walker to increase from $k$ to $k+1$. Alternatively, $\theta(N-k, N)$ is the time needed to visit a new site among the $N-k$ unvisited sites, once $k$ sites have been visited. The following exact expression then holds:

$$
\tau(M, N)=\sum_{k=N-M+1}^{N-1} \theta(k, N)
$$

The exact determination of the statistical properties of this random variable can seem out of reach because a priori $\theta(k, N)$ depends on the entire random trajectory until time $\tau(N-k, N)$. The key hypothesis is then to assume that the random variables $\theta(k, N)$ are in fact independent asymptotically in the large- $N$ limit. This hypothesis will be verified numerically for all tested non-compact random walks, and will furthermore allow the retrieval of exact results known so far for regular random walks. In this regime of $N$ large the distribution of $\theta(k, N)$ can be obtained explicitly and enables the determination of the full distribution of the partial cover time, which, as we show in the Supplementary Information, finally takes the universal form

$$
P(x)=\frac{1}{p !} \exp \left(-(p+1) x-\mathrm{e}^{-x}\right)
$$

valid in the limit $N, M \rightarrow \infty$ with $p \equiv N-M$ fixed (implying in particular $p / N \rightarrow 0$ ). Here the rescaled variable $x \equiv \tau /\langle T\rangle-\ln N$ involves the mean $\langle T\rangle$ of the global first-passage time, defined as the mean first-passage time to a given target site averaged over all starting sites. This constitutes the central result of this paper. Its derivation relies on the fact that the distribution of the global firstpassage time $T$ to a given target site is asymptotically an exponential

${ }^{1}$ Laboratoire de Physique Théorique de la Matière Condensée, UMR 7600 CNRS /UPMC, 4 Place Jussieu, 75255 Paris Cedex, France. ${ }^{2}$ Laboratoire Jean

Perrin, UMR 8237 CNRS /UPMC, 4 Place Jussieu, 75255 Paris Cedex, France. *e-mail: benichou@lptmc.jussieu.fr; voiturie@lptmc.jussieu.fr 


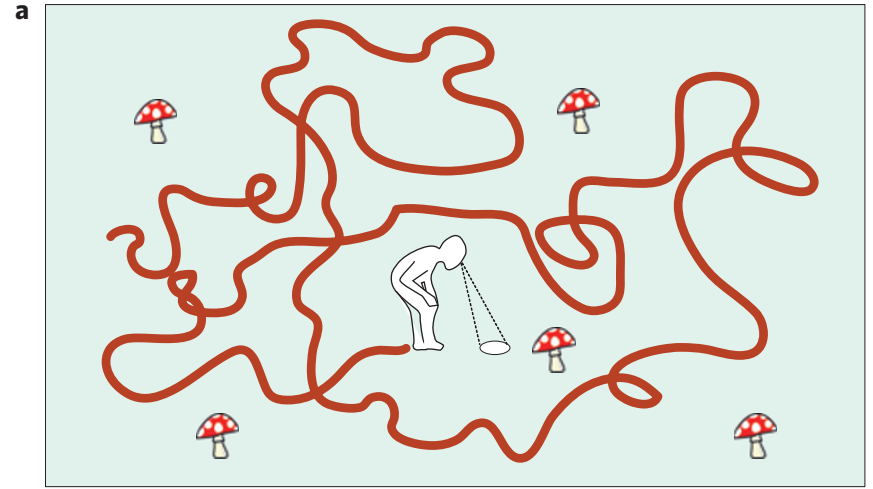

b
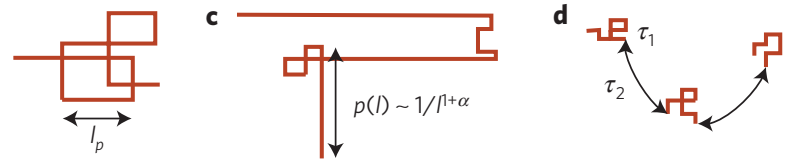

Persistent walk
Intermittent walk

Figure 1 | How long does it take to exhaustively explore a given domain? This quantity defines the cover time of the domain. $\mathbf{a}$, An example of exhaustive search: the time needed to find all mushrooms and exhaust a given area, with no prior knowledge of their distribution in space, is the cover time of the domain. In this paper we also consider the time needed to visit any $M$ sites of the domain, defined as the partial cover time, as well as the time needed to visit $M$ given sites of the domain chosen at random, defined as the random cover time. b-d, Examples of optimizable search processes (see Methods): persistent random walks (b), Lévy walks (c) and intermittent random walks (d). For all these search processes, we show that the distribution of the cover time takes a universal form, and that the mean cover time can be minimized.

of mean $\langle T\rangle$ for non-compact random walks ${ }^{29,30}$. In addition, we assumed that $\langle T\rangle$ is independent of the target site, which is exact for domains with periodic boundary conditions, and in practice

satisfied well for any domain shape in the case of non-compact random walks, as was checked numerically.

Several comments are in order. The result of equation (1) unveils the universal dependence of the distribution of the partial cover time on both the random walk process-through only the global mean first-passage time to a single target-and the geometry of the domain - through only its volume $N$ (using that $\langle T\rangle$ asymptotically depends on the geometry only through $N$; refs 18,21). Equation (1) also reveals a deep connection with order statistics, already pointed out in the mathematical literature for regular random walks 9 Indeed, equation ( 1$)$ is the limit distribution of the $(p+1)$ th largest among $N$ independent identically distributed random variables in the large- $N$ regime (Gumbel universality class). In fact, in the case $M=N$, the full cover time can easily be seen as the largest among the first-passage times $t_{i}$ of the searcher to site $i$, where $i \in \llbracket 1, N \rrbracket$ covers all sites of the domain. Equation (1) indeed yields in this case $(p=0)$ the classical Gumbel law. More generally, the partial cover time $\tau(N-p, N)$ can be seen as the $(p+1)$ th largest among the $\left\{t_{i}\right\}_{i \in \llbracket 1, N \rrbracket}$. What we find here is that, in the case of noncompact exploration, the $\left\{t_{i}\right\}_{i \in \llbracket 1, N \rrbracket}$ are asymptotically independent in the large- $N$ regime. Furthermore, equation (1) provides, in particular, an explicit determination of the mean partial cover time $\langle\tau\rangle \sim\langle T\rangle\left(\ln N-\Psi^{(0)}(p+1)\right)$ in the large- $N$ regime, where $\Psi^{(n)}$ denotes the polygamma function of order $n$, and which is in agreement with exact results obtained for the full cover time in the particular case of Brownian walks on periodic lattices ${ }^{9,23}$. Beyond the mean, the variance of the partial cover time can be determined and is given asymptotically by $\sigma^{2} \sim \Psi^{(1)}(p+1)\langle T\rangle^{2}$. This corresponds to a reduced variance $\sigma^{2} /\langle\tau\rangle^{2} \sim \Psi^{(1)}(p+1) / \ln ^{2} N$, which shows that the amplitude of the relative fluctuations slowly decays with the domain size. Finally, the distribution of related observables can be readily deduced from this approach, and depends on the search process only through the global mean first-passage time $\langle T\rangle$ to a given target in the domain for a single searcher. For example the distribution of the random cover time, defined above as the time needed to visit $M$ given sites of a domain of $N$ sites $^{27}$, is obtained in the limit $M, N \gg 1$ by taking $p=0$ in equation (1) (Gumbel law), with the new rescaled variable $x \equiv \tau /\langle T\rangle-\ln M$ (see Supplementary Information). In a

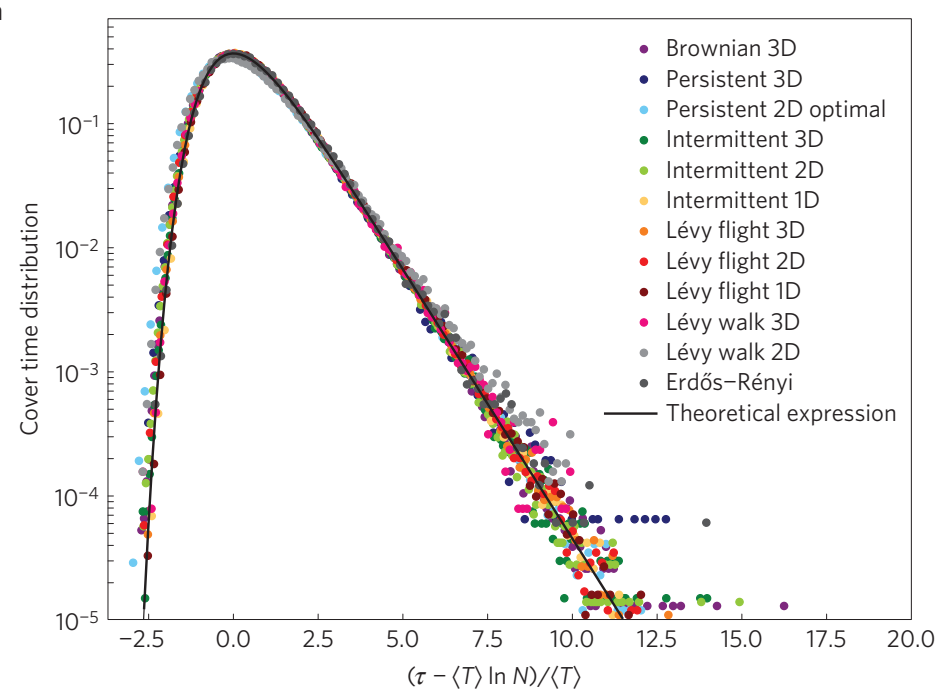

b

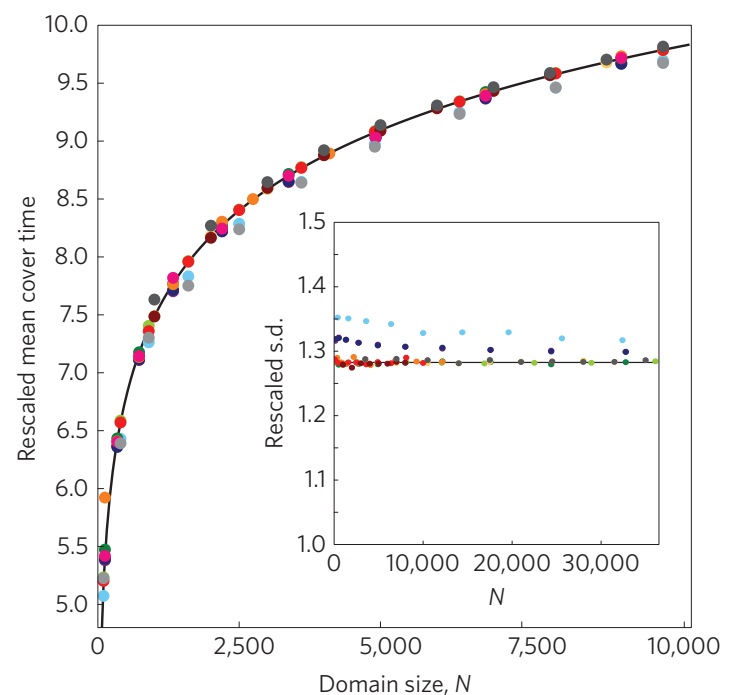

Figure 2 | Universal distribution of the full cover time $(M=N)$ for non-compact search processes. a, Distribution of the rescaled cover time for various non-compact search processes. All data collapse to a universal master curve defined by equation (1) with $p=0$ (solid line). $\mathbf{b}$, Mean cover time (rescaled by the global mean first-passage time) as a function of the domain size $N$. The solid line gives the theoretical prediction $\ln N-\Psi^{(0)}(1)=\ln N+\gamma$, where $\gamma$ denotes the Euler constant. Inset: standard deviation of the cover time (rescaled by the global mean first-passage time) as a function of the domain size $N$. The solid line gives the theoretical prediction $\Psi^{(1)}(1)=\pi^{2} / 6$. For all panels, domain sizes and all parameters defining the search processes are listed in Supplementary Information. 


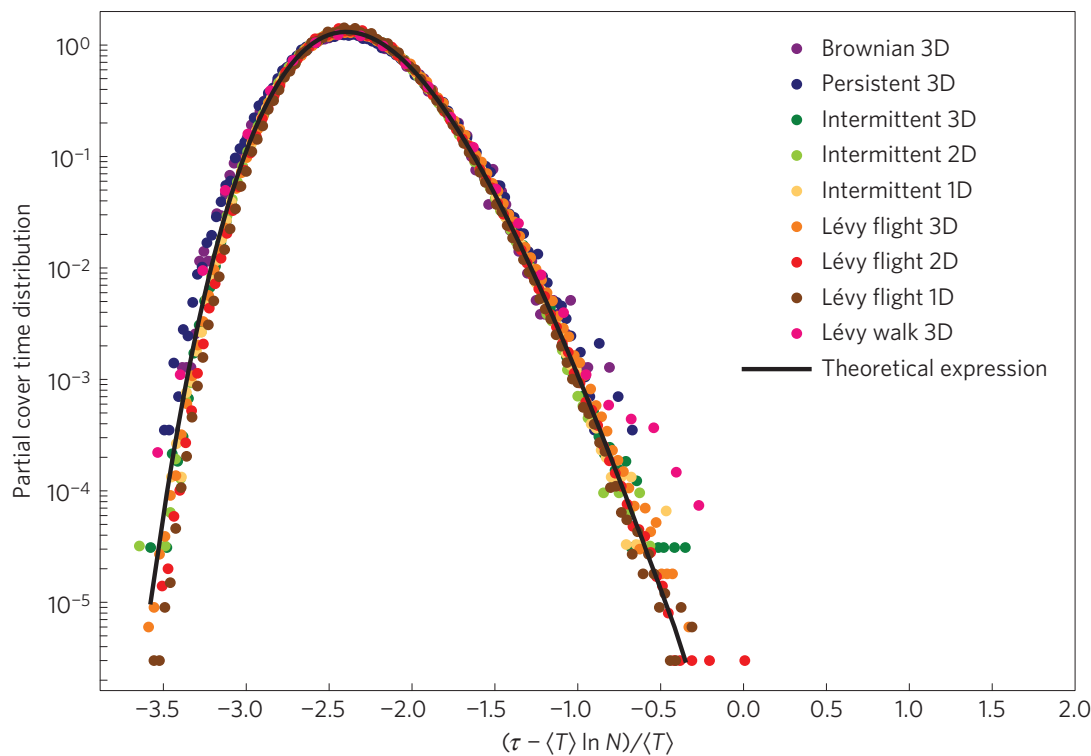

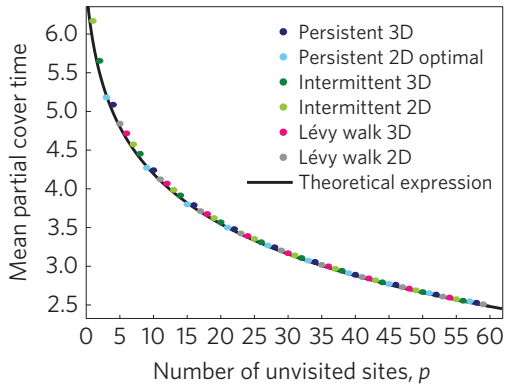

C

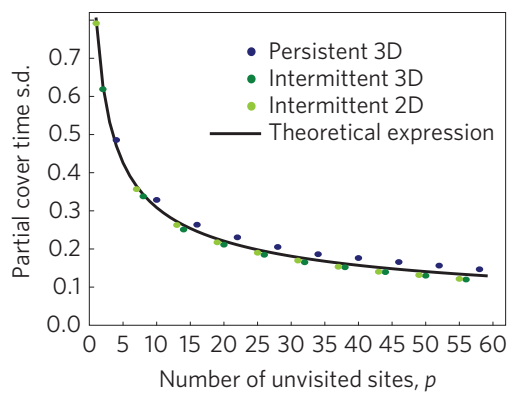

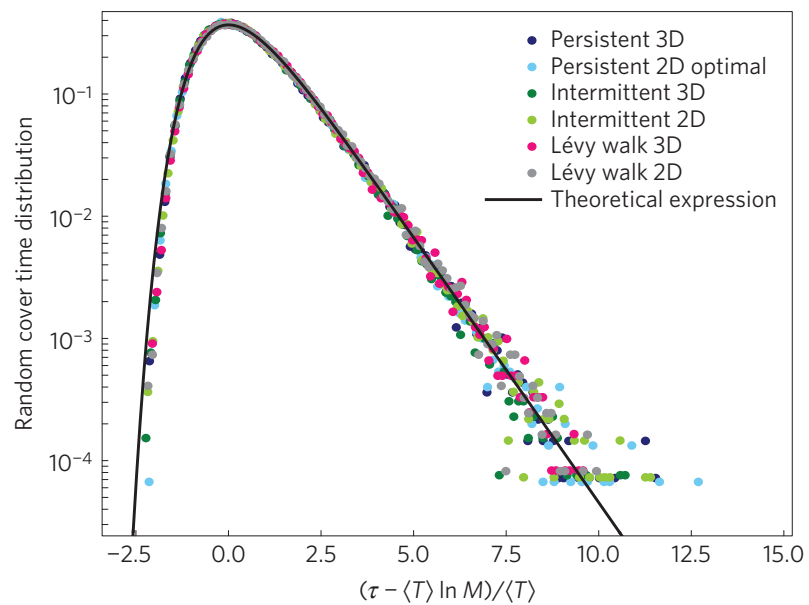

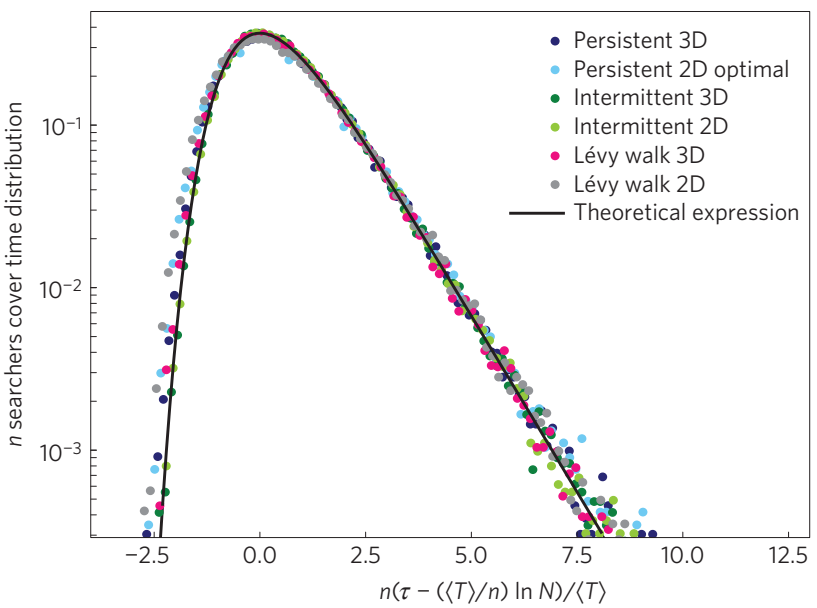

Figure 3 | Universal distribution of cover time type observables for non-compact search processes. a, Distribution of the rescaled partial cover time for various non-compact search processes. All data collapse to a universal master curve defined by equation (1), here for $p \equiv N-M=10$ unvisited sites (solid line). b, Mean partial cover time (rescaled by the global mean first-passage time) as a function of $p$ for $N$ fixed. The solid line gives the theoretical prediction $\ln N-\Psi^{(0)}(p+1)$. c, Standard deviation of the partial cover time (rescaled by the global mean first-passage time) as a function of $p$ for $N$ fixed. The solid line gives the theoretical prediction $\Psi^{(1)}(p+1)$. d. Distribution of the rescaled random cover time for various non-compact search processes. All data collapse to a universal master curve defined by equation (1) with $p=0$, here for $M=20$ randomly chosen given sites to visit (solid line). e, Distribution of the rescaled full cover time for various non-compact search processes with $n$ independent searchers. All data collapse to a universal master curve defined by equation (1) with $p=0$, here for $n=10$ searchers (solid line). For all panels, domain sizes (all such that $N \gg 1$ ) and all parameters defining the search processes are listed in Supplementary Information.

turn, in the important case where $n$ searchers explore the domain simultaneously, the distribution of the partial cover time is also given by equation (1), with the new rescaled variable $x \equiv n \tau /\langle T\rangle-\ln N$.

We now confirm these analytical results by Monte Carlo simulations of most of the models of random search strategies invoked in the literature: Brownian random walks, persistent random walks, Lévy flights and Lévy walks, intermittent random walks, and random walks on complex networks. Figures 2 and 3 reveal excellent quantitative agreement between the analytical predictions and the numerical simulations. The prediction of equation (1) unambiguously captures the mean, variance and entire distribution of partial and full cover times (Figs 2 and 3), as well as random cover times and cover times for $n$ searchers (Fig. 3), as shown by the data collapse of the numerical simulations. We emphasize that the very different nature of these examples demonstrates that the range of applicability of our approach, which mainly relies on the non-compact property of the random trajectory of the searcher, is wide. Note that even for two-dimensional (2D) persistent random walks, which are in fact marginally compact, both the mean and variance of cover times are quantitatively predicted by our approach in the large-domain-size limit, provided that the persistence length minimizes the global mean first-passage time for a target $\langle T\rangle$, as analysed in ref. 17 .

In practice, the question of minimizing the cover time to optimize the efficiency of the search process is crucial. Our analysis reveals that the mean cover time (either random or partial) is minimized exactly when the global mean first-passage time $\langle T\rangle$ for a single target is minimized (see Fig. 4). In particular, in the case of persistent random walks and intermittent random walks, which have been shown to minimize $\langle T\rangle$ (either by tuning the persistence length of the persistent random walk or the duration of the scanning phase of the intermittent random walk), we find that mean cover times can 

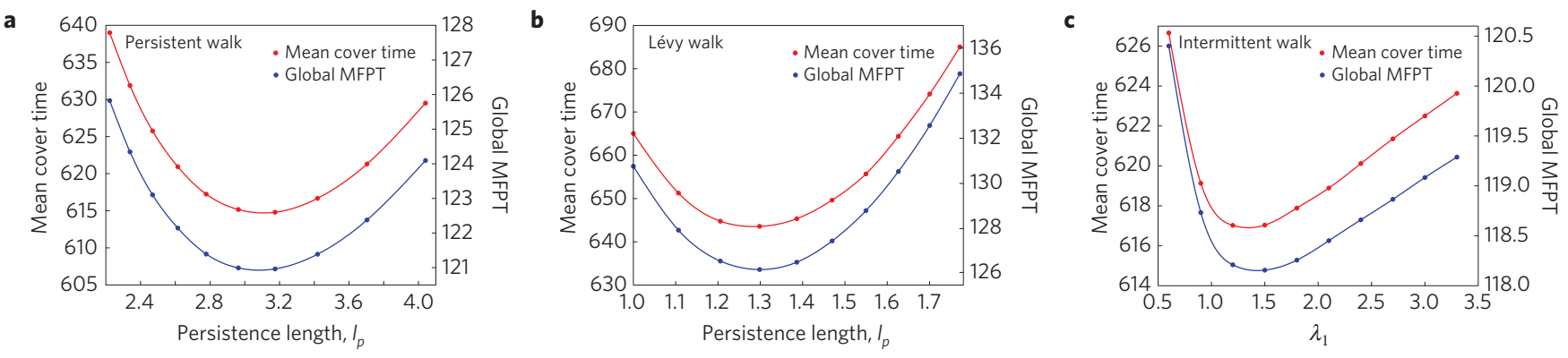

Figure 4 | The mean full cover time and the mean search time for a single target can be minimized by the same optimal strategy. The mean full cover time and the global mean first-passage time to a single target are plotted as a function of the persistence length for persistent random walks (a, here in $2 \mathrm{D}$, $N=100$ ) and Lévy walks ( $\mathbf{b}$, here in $2 \mathrm{D}$ with $\alpha=1.8, N=100$ ), and as a function of the switching rate $\lambda_{1}$ (see Methods and Supplementary Information) for intermittent random walks (c, here with $\rho=1, \lambda_{2}=0.8, N=100$ ).

also be minimized. For Lévy walks, the celebrated optimization of the target encounter rate for a Lévy index $\alpha \simeq 1$ (defined through the jump length distribution $p(l) \propto l^{-\alpha-1}$ for $l$ large) has been obtained and discussed only for a distribution of infinitely many so-called revisitable targets (which reappear at the same position after being found $)^{12}$. In contrast, the global mean first-passage time to a single target in confinement, which by definition involves a non-revisitable target, has been left aside. In fact, we find that $\langle T\rangle$ (see also ref. 17), and therefore the mean cover time, can be minimized by adjusting the persistence length for all $\alpha>1$ (see Fig. 4). This optimal strategy, which does not require revisitable targets, is therefore very different from the above-mentioned optimal strategy obtained for infinitely many revisitable targets. All together, these results shed new light on the role of persistent, intermittent and Lévy strategies in the optimization of search processes, and clearly points towards their robustness.

\section{Methods}

Methods and any associated references are available in the online version of the paper.

Received 20 April 2015; accepted 25 June 2015; published online 3 August 2015

\section{References}

1. Aldous, D. On the time taken by random walks on finite groups to visit every state. Z. für Wahrscheinlichkeitstheor. Verwandte Geb. 62, 361-374 (1983).

2. Heuzé, M. L. et al. Migration of dendritic cells: Physical principles, molecular mechanisms, and functional implications. Immunol. Rev. 256, 240-254 (2013).

3. Viswanathan, G. M., Raposo, E. P. \& da Luz, M. G. E. Lévy flights and superdiffusion in the context of biological encounters and random searches. Phys. Life Rev. 5, 133-150 (2008).

4. Bénichou, O., Loverdo, C., Moreau, M. \& Voituriez, R. Intermittent search strategies. Rev. Mod. Phys. 83, 81-129 (2011).

5. Vergassola, M., Villermaux, E. \& Shraiman, B. I. Infotaxis as a strategy for searching without gradients. Nature 445, 406-409 (2007).

6. Brummelhuis, M. J. A. M. \& Hilhorst, H. J. Covering of a finite lattice by a random walk. Physica A 176, 387-408 (1991).

7. Dembo, A., Peres, Y., Rosen, J. \& Zeitouni, O. Cover times for Brownian motion and random walks in two dimensions. Ann. Math. 160, 433-464 (2004).

8. Ding, J. On cover times for 2D lattices. Electron. J. Probab. 17, 45 (2012).

9. Belius, D. Gumbel fluctuations for cover times in the discrete torus. Probab. Theory Relat. Fields 157, 635-689 (2013).

10. Shlesinger, M. F. \& Klafter, J. in Lévy Walks vs Lévy Flights (eds Stanley, H. E. \& Ostrowski, N.) 279-283 (Springer, 1986).

11. Viswanathan, G. M. et al. Levy flight search patterns of wandering albatrosses. Nature 381, 413-415 (1996)

12. Viswanathan, G. M. et al. Optimizing the success of random searches. Nature 401, 911-914 (1999).
13. Metzler, R. \& Klafter, J. The random walk's guide to anomalous diffusion: A fractionnal dynamics approach. Phys. Rep. 339, 1-77 (2000).

14. Lomholt, M. A., Tal, K., Metzler, R. \& Joseph, K. Lévy strategies in intermittent search processes are advantageous. Proc. Natl Acad. Sci. USA 105, 11055-11059 (2008)

15. Benichou, O., Coppey, M., Moreau, M., Suet, P.-H. \& Voituriez, R. Optimal search strategies for hidden targets. Phys. Rev. Lett. 94, 198101 (2005).

16. Oshanin, G., Wio, H. S., Lindenberg, K. \& Burlatsky, S. F. Intermittent random walks for an optimal search strategy: One-dimensional case. J. Phys.: Condens. Matter 19, 065142 (2007).

17. Tejedor, V., Voituriez, R. \& Bénichou, O. Optimizing persistent random searches. Phys. Rev. Lett. 108, 088103 (2012).

18. Condamin, S., Benichou, O., Tejedor, V., Voituriez, R. \& Klafter, J. First-passage times in complex scale-invariant media. Nature 450, 77-80 (2007).

19. Redner, S. A Guide to First-Passage Processes (Cambridge Univ. Press, 2001).

20. Bénichou, O., Chevalier, C., Klafter, J., Meyer, B. \& Voituriez, R. Geometry-controlled kinetics. Nature Chem. 2, 472-477 (2010).

21. Bénichou, O. \& Voituriez, R. From first-passage times of random walks in confinement to geometry-controlled kinetics. Phys. Rep. 539, 225-284 (2014).

22. Bray, A. J., Majumdar, S. N. \& Schehr, G. Persistence and first-passage properties in nonequilibrium systems. Adv. Phys. 62, 225-361 (2013).

23. Aldous, D. An introduction to covering problems for random walks on graphs. J. Theor. Probab. 2, 87-89 (1989).

24. Weiss, G. \& Shlesinger, M. On the expected number of distinct points in a subset visited by ann-step random walk. J. Stat. Phys. 27, 355-363 (1982).

25. Burov, S. \& Barkai, E. Weak subordination breaking for the quenched trap model. Phys. Rev. E 86, 041137 (2012).

26. Yokoi, C. S. O., Hernández-Machado, A. \& Ramírez-Piscina, L. Some exact results for the lattice covering time problem. Phys. Lett. A 145, 82-86 (1990).

27. Nemirovsky, A. M., Mártin, H. O. \& Coutinho-Filho, M. D. Universality in the lattice-covering time problem. Phys. Rev. A 41, 761-767 (1990).

28. Hughes, B. Random Walks and Random Environments (Oxford Univ. Press, 1995).

29. Meyer, B., Chevalier, C., Voituriez, R. \& Bénichou, O. Universality classes of first-passage-time distribution in confined media. Phys. Rev. E 83, 051116 (2011).

30. Bénichou, O., Chevalier, C., Meyer, B. \& Voituriez, R. Facilitated diffusion of proteins on chromatin. Phys. Rev. Lett. 106, 038102 (2011).

\section{Acknowledgements}

O.B. was supported by ERC grant FPTOpt-277998.

\section{Author contributions}

All authors contributed equally to this work.

\section{Additional information}

Supplementary information is available in the online version of the paper. Reprints and permissions information is available online at www.nature.com/reprints.

Correspondence and requests for materials should be addressed to O.B. or R.V.

\section{Competing financial interests}

The authors declare no competing financial interests. 


\section{Methods}

The definition of random search processes analysed in the text are as follows (see Supplementary Information for details): Brownian random walks constitute the most striking example of a random search process, which is known to be non-compact in dimension $D=3$, and marginally compact for $D=2$. Here we consider nearest-neighbour random walks on a periodic lattice of $N$ sites. Lévy flights have been shown to play an important role in random search problems ${ }^{13}$. We consider here discrete Lévy flights of index $\alpha$ on 1D, 2D and 3D periodic lattices of size $N$, characterized by a probability distribution to perform a jump of size $l$ that obeys $p(l) \propto l^{-\alpha-1}$ for $l$ large. Beyond classical Euclidean spaces, many examples of random walks on complex networks, whose relevance to extremely various fields is now unanimously recognized, are non-compact. Here we consider the emblematic Erdös-Rényi networks ${ }^{31}$. Persistent random walks provide a minimal example of a search process with memory, which is encoded in the persistence length, defined as the mean number of successive steps performed in a given direction. They have been shown to lead to a minimization of the mean search time for a single target, and therefore play a prominent role in the optimization of search processes ${ }^{17}$. We consider here 2D and 3D discrete persistent random walks on a periodic lattice of $N$ sites. As opposed to Lévy flights, which can have arbitrary large velocities, Lévy walks ${ }^{10}$ have a constant speed, and can be seen as an extension of persistent random walks, for which the distribution of the number of successive steps is not exponential, but power-law distributed. They have been shown to be optimizable and have been extensively invoked in the context of animal behaviour ${ }^{12}$. We consider here 2D and 3D discrete Lévy walks on a periodic lattice of $N$ sites. Last, we consider the case of well-hidden targets, for which it can be assumed that moving and searching are incompatible. In this case, the search strategy is intermittent ${ }^{4}$. Technically, we focus here on a continuous time two-state searcher moving on a 1D, 2D or 3D periodic lattice of $N$ sites $^{32}$. In the slow reactive state 1 , the searcher performs a regular nearest-neighbour random walk with jump rate $\rho$, and actually visits the corresponding sites. With rate $\lambda_{1} \equiv 1 / \tau_{1}$, the searcher switches to a fast and non-reactive state 2 , which enables a uniform relocalization in the domain, but during which no sites are visited. The searcher then switches to state 1 with rate $\lambda_{2} \equiv 1 / \tau_{2}$.

\section{References}

31. Albert, R. \& Barabasi, A.-L. Statistical mechanics of complex networks. Rev. Mod. Phys. 74, 47-97 (2001)

32. Benichou, O., Moreau, M., Suet, P.-H. \& Voituriez, R. Intermittent search process and teleportation. J. Chem. Phys. 126, 234109 (2007). 